\title{
Evidencia Complementaria para la Validación de la Escala SRAS de Altruismo en Colombia
}

\author{
Complementary Evidence for the Validation of the Altruism SARS Scale in \\ Colombia
}

\author{
Orlando Scoppetta ${ }^{1}$, Carlos Pardo Adames ${ }^{2}$, David Aguilar-Pardo ${ }^{3}$ y Idaly Barreto ${ }^{4}$
}

\begin{abstract}
Resumen
Se asume que la tendencia a ayudar sin esperar recompensas, es una característica estable que puede ser medida utilizando instrumentos desarrollados por medios psicométricos. La Escala de Auto-reporte de Altruismo (SRAS) se usa en distintos contextos culturales y fue adaptada para Colombia, en una población de estudiantes universitarios. El objetivo de esta investigación fue obtener evidencia complementaria para la validación de la SRAS en Colombia.. La SRAS adaptada para Colombia se aplicó a 244 personas de diferentes niveles educativos, y se calcularon los índices del Modelo de Rasch. Hubo funcionamiento diferencial en un ítem. Los indicadores mostraron buen ajuste de la prueba. Se encontraron tres puntos de anclaje que permitieron dividir a los participantes en cuatro grupos de desempeño. Aunque se encontró evidencia acerca de la utilidad de la prueba para medir altruismo, se sugiere una adaptación más profunda al contexto nacional.
\end{abstract}

Palabras clave: comportamiento prosocial, ayuda, Modelo de Rasch, psicometría, funcionamiento diferencial de ítems

\begin{abstract}
It is assumed that the tendency to help others without expecting rewards is a stable characteristic that can be measured using instruments developed by psychometric means. This is the concept in the base of Altruism Self Reporting Scale (SARS), which is used in different cultural contexts and was adapted for Colombia, in a sample of university students. The study aimed to get complementary evidence for the validation of SARS in Colombia. The adapted SARS was applied to 244 people of different educational levels, and the indices of the Rasch Model were calculated. There was differential operation in one item. The indicators showed good adjustment of the test. Three anchoring points were found that allowed the participants to be divided into four performance groups. Although evidence was found about the usefulness of the test to measure altruism, a deeper adaptation to the national context is suggested.
\end{abstract}

Keywords: prosocial behavior, helping, Rasch model, psychometry, differential item functioning

\footnotetext{
${ }^{1}$ Psicólogo. Magister en Estudios de Población. Magister en Psicología. PhD. En Psicología. Universidad Católica de Colombia. Avenida Caracas \#46-22, Colombia. Tel.: (+571) 3277300. Correo: oscoppetta18@ucatolica.edu.co

${ }^{2}$ Psicólogo. Magister en Educación. Universidad Católica de Colombia. Estudiante del Doctorado en Psicología. Avenida Caracas \#46-22, Colombia. Tel. (+571) 3277300. Correo: capardo@ucatolica.edu.co

${ }^{3}$ Biólogo. Magister en Fisiología. Universidad Católica de Colombia. Docente Facultad de Psicología. Avenida Caracas \#46-22. Tel.: (+571) 3277300. Correo: draguilar@ucatolica.edu.co

${ }^{4}$ Psicóloga. Doctora en Psicología. Universidad Católica de Colombia. Decana de Psicología. Avenida Caracas \#46-22. Tel.: (+571) 3277300. Correo: mibarreto@ucatolica.edu.co

Revista Iberoamericana de Diagnóstico y Evaluación - e Avaliação Psicológica. RIDEP · No58 · Vol.1 · 47-55 · 2021

ISSN: 1135-3848 print /2183-6051online
} 


\section{Introducción}

El altruismo es una forma de comportamiento prosocial que satisface la necesidad de otra persona y se distingue por el sacrificio y la no obtención de una ganancia que pueda ser identificada de manera objetiva (Auné et al., 2014; Kawamura \& Kusumi, 2018; Liu et al., 2016). En el análisis del altruismo confluyen diferentes disciplinas como la biología, la sociología y la psicología (Ramsey, 2016). Un capítulo importante de la discusión desde estas disciplinas tiene que ver con esclarecer si lo que se llama altruismo es una característica estable, que puede medirse en cualquier grupo de personas, o no es más que la consecuencia eventual del intercambio social (Chaplain, 2017; Leimgruber, 2018; Santamaría-García et al., 2018). Al respecto, hay evidencia de que el comportamiento altruista toma diferentes formas dependiendo del contexto y las características de los actores (Wittek \& Bekkers, 2015). Por ejemplo, Ovejero Bernal (2007) plantea que el altruismo puede variar de acuerdo con: la edad, basado en estudios que muestran cómo el altruismo crece entre los seis y los doce años; el sexo, dado que las mujeres tienden a ser más sociables; la personalidad, puesto que determinados rasgos pueden predecir mejor la conducta altruista; y el estado de ánimo, dado que las personas con mejor humor tienden a ser sistemáticamente más dispuestas a la ayuda.

Aunque la conducta altruista pueda variar de acuerdo con la configuración de distintos factores, se postula que el altruismo es una característica que se mantiene constante y permite que algunas personas sean percibidas como más altruistas que otras. Sobre esta base, Rushton, Chrisjohn y Fekken (1981), desarrollaron la Escala de Autoreporte de Altruismo (SRAS, por sus siglas en inglés), que ha sido utilizada en investigaciones sobre altruismo y su relación con otras variables, en distintos lugares del mundo (Lilienfeld et al., 2016). Adicionalmente, esta escala ha sido adaptada a diversos entornos culturales, como en el caso de la versión india (Khanna et al., 1993) y la versión china (Chou, 1996).

En Colombia, Aguilar-Pardo y MartínezCotrina (2016) adaptaron el lenguaje de la SRAS al contexto nacional y analizaron sus propiedades psicométricas. Asumieron que la escala está constituida por un único factor y encontraron una fiabilidad de .82. En principio Rushton et al., (1981) no plantean la existencia de diversos factores en su escala, pero Chaplain (2017) encuentra que los ítems de la prueba conforman tres factores que agrupan los reactivos de acuerdo con el riesgo que implica la conducta altruista. Así, por ejemplo, haber donado ropa o bienes a una entidad de caridad, se considera una conducta dentro del factor de menos riesgo; ayudar a empujar el automóvil de un extraño se incluye dentro de los ítems de riesgo mediano; y llevar a un extraño en el automóvil propio haría parte del factor correspondiente a comportamientos que implican riesgo más alto.

De acuerdo con lo anterior, contar con una escala específica para medir altruismo, es de utilidad para establecer con mayor validez y precisión los factores asociados; las diferencias entre grupos de personas que se comportan de manera altruista con más frecuencia (Guarnaccia etal., 2016), o las predicciones de las consecuencias de tales comportamientos (Arnocky et al., 2017). En este marco, la adaptación de una prueba como la SRAS, es un proceso complejo que implica diferentes aspectos que van desde el aseguramiento de la validez, el análisis de la confiabilidad y el estudio de la capacidad de la prueba para clasificar a las personas de acuerdo con el grado del atributo medido (DeVellis, 2017).

Entre las distintas aproximaciones psicométricas para la adaptación y validación de una prueba, los análisis basados en el modelo de Rasch, permiten avanzar sobre la base de instrumentos ya existentes debido a la información que ofrece este tipo de abordaje acerca del comportamiento de la prueba en cuestión (Abello-Luque et al., 2017; Jerosch-Herold et al., 2017) facilitando la optimización de la prueba así como la discriminación de sujetos a lo largo de las calificaciones de las escalas (Bartholomeu et al., 2016). El modelo de Rasch, se centra en que la probabilidad de una respuesta de un evaluado a un ítem se puede describir como una función simple de la habilidad de la persona (su desempeño en el constructo) y la dificultad del ítem. De esta manera, se contribuye a mejorar la calidad técnica de una prueba ya que pueden detectar preguntas inadecuadas para el propósito de la medición (Molenaar, 1995). 
Aunque en América Latina se usan diferentes instrumentos para evaluar comportamientos prosociales, como la Escala de Responsabilidad Social y Participación en Actividades Cívicas (Berger et al., 2016) o la Escala de Creencias Normativas sobre la Prosocialidad (Velásquez et al., 2004), se optó por la SRAS, debido al antecedente de adaptación en Colombia (AguilarPardo \& Martínez-Cotrina, 2016) y, teniendo en cuenta que, la adaptación y la validación de una prueba psicométrica son procesos continuos, se deben concatenar esfuerzos que apunten al mejoramiento progresivo de instrumentos para estimar cada vez mejor los constructos de interés.

En el presente caso, si bien la SRAS muestra un buen comportamiento psicométrico en todos los idiomas en los que se ha aplicado, incluyendo la adaptación hecha para Colombia, es pertinente seguir trabajando en su desarrollo. Uno de los caminos posibles para esto es la aplicación del modelo de Rasch, el cual permite evaluar establecer valores de precisión de la medición de cada ítem. En futuros trabajos se podría apuntar al mejoramiento de este instrumento desde otras perspectivas metodológicas.

Desde esta aproximación el objetivo de este estudio es obtener evidencia complementaria para la validación de la SRAS en Colombia, con índices obtenidos desde la perspectiva de la Teoría de Respuesta al Ítem, en particular el Modelo de Rasch, tanto para el cuestionario en su totalidad como para cada uno de los ítems.

\section{Método}

Este estudio es de tipo instrumental, dirigido a analizar las características psicométricas de la SRAS, mediante la aplicación del modelo de Rasch (Ato et al., 2013).

\section{Participantes}

El estudio se llevó a cabo con 244 participantes seleccionados no probabilísticamente, por conveniencia, con la siguiente procedencia: 49 estudiantes de una institución de educación básica y media; 110 estudiantes de cuatro instituciones de educación superior y 85 personas mayores de 40 años, no estudiantes al momento de la investigación. De la totalidad de participantes 133 fueron mujeres $(54.5 \%)$ y 111 hombres $(45.6 \%)$.
La edad promedio fue de 32.4 años (DT=14.8); 32.4 para los hombres y 32.5 para las mujeres con un rango para todo el grupo entre los 14 y los 67 años. Se escogió la muestra así considerando que el constructo es transversal a las subpoblaciones y que el estudio de autores, solamente se hizo con estudiantes universitarios.

La presente investigación se ajusta a la legislación colombiana vigente promulgada en la resolución número 8430 de 1993 la cual dicta las normas científicas, técnicas y administrativas para la investigación en salud, dentro de la cual se clasifica a los estudios sin manipulación de la conducta, como investigaciones sin riesgo o de riesgo mínimo. También se ajustó a la práctica de investigaciones en psicología, promulgadas en la ley 1090 de 2006.

Se contó con el consentimiento del encuestado y la opción de no participar en la investigación. Las bases de datos a utilizar no incluyen información que permita identificar a los participantes.

\section{Instrumentos}

Se utilizó la Escala de Auto-reporte de Altruismo (SRAS), adaptada por Aguilar-Pardo y Martínez-Cotrina (2016), la cual consta de 20 ítems que incluyen conductas de ayuda a desconocidos, a conocidos y a instituciones benéficas. Cuenta con una escala de respuesta uniforme compuesta por las opciones: nunca, una vez, más de una vez, frecuentemente y muy frecuentemente. En la investigación original de creación de la escala, se reportaron valores del alpha de Cronbach entre .78 y .87 en cinco muestras estudiadas (Rushton et al., 1981). Por su parte, Aguilar-Pardo y MartínezCotrina (2016) calcularon este indicador en .82.

\section{Procedimiento}

El estudio se realizó en varias fases para determinar la calidad técnica del instrumento.

FASE 1: Administración del cuestionario. El cuestionario de altruismo se administró como un cuestionario de auto reporte, en el que se siguió la arquitectura de cuatro procesos según Mislevy et al. (2003). Proceso 1: Presentación. Se entregó el cuestionario en formato de lápiz y papel para ser diligenciado por los sujetos seleccionados. Proceso 2: procesamiento de respuestas. Las respuestas se tradujeron en valores ordenados (de 1 a 5) que identifican la frecuencia de realización 
Tabla 1. DIF según sexo Femenino - Masculino

\begin{tabular}{|c|c|c|c|c|c|}
\hline Ítem & Dificultad Femenino & Dificultad masculino & Diferencia & Regresión Logística P & Mantel P \\
\hline ALT01 & .70 & -.20 & .90 & .00 & .00 \\
\hline ALT02 & -.82 & -.94 & .12 & .42 & .58 \\
\hline ALT03 & .29 & .45 & -.16 & .26 & .20 \\
\hline ALT04 & .42 & .55 & -.12 & .39 & .74 \\
\hline ALT05 & -.48 & -.37 & -.10 & .47 & .60 \\
\hline ALT06 & -.48 & -.27 & -.21 & .14 & .64 \\
\hline ALT07 & .85 & 1.05 & -.20 & .22 & .57 \\
\hline ALT08 & .73 & .73 & .00 & 1.00 & .64 \\
\hline ALT09 & -.38 & -.26 & -.12 & .39 & .12 \\
\hline ALT10 & -.90 & -.98 & .07 & .61 & .33 \\
\hline ALT11 & -.48 & -.30 & -.17 & .21 & .29 \\
\hline ALT12 & 1.67 & 1.37 & .30 & .14 & .10 \\
\hline ALT13 & -.25 & -.43 & .17 & .22 & .31 \\
\hline ALT14 & .48 & .67 & -.19 & .20 & .21 \\
\hline ALT15 & .06 & .10 & -.05 & .74 & .28 \\
\hline ALT16 & -1.04 & -1.08 & .04 & .78 & .97 \\
\hline ALT17 & .56 & .87 & -.32 & .04 & .03 \\
\hline ALT18 & -.50 & -.48 & -.02 & .87 & .98 \\
\hline ALT19 & -.94 & -.94 & .00 & 1.00 & .88 \\
\hline ALT20 & .58 & .41 & .16 & .26 & .47 \\
\hline
\end{tabular}

de las tareas planteadas en el cuestionario. Proceso 3: acumulación de evidencia. Se utilizó el software Winsteps ${ }^{\circledR}$, versión 4.0.1, que procesa los datos con el modelo de Rasch, con el método de Andrich para escalas de valoración (Linacre, 2017). Proceso cuatro: análisis de resultados.

FASE 2: diseño y depuración de la base de datos. En esta fase se estableció que la base de datos debería contener una identificación generalizada del evaluado, su edad, sexo y los valores de las respuestas a cada ítem, correspondientes a la valoración asignada a cada alternativa de respuesta. La base de datos se depuró siguiendo los planteamientos de autores, con base en el desajuste de los evaluados en relación con el modelo. En este caso se utilizó el criterio de no incluir a evaluados con valores de ajuste iguales o superiores a 2.0 (Linacre, 2017), ya que pueden introducir distorsión o degradar el sistema de medición (18 personas identificadas).

FASE 3: procesamiento y análisis de resultados. En esta fase se realizó el procesamiento de datos con el software Winsteps ${ }^{\circledR}$ y se analizaron los indicadores obtenidos, relacionados con la calidad técnica del instrumento. En un primer momento se realizó un análisis de Funcionamiento Diferencial de Ítems (DIF por su sigla en inglés), utilizando los métodos Mantel-Haenszel y regresión logística implementados por el software Winsteps ${ }^{\circledR}$. Con base en la información ya depurada de datos de los participantes e ítems se procedió a la producción de los indicadores de calidad del instrumento.
De acuerdo con Spector (1992), la SRAS se puede clasificar como una escala de valoración tipo Likert, por lo que el valor de la confiabilidad se estableció con base en dos indicadores: confiabilidad de Rasch y el índice de separación (Gliem \& Gliem, 2003; Linacre, 2017), que son medidas de consistencia interna (Linacre, 1997, 2017).

La unidimensionalidad de un instrumento de evaluación es uno de los supuestos del Modelo de Rasch y se define como el rasgo latente que da cuenta o subyace al desempeño de las personas en los ítems de un cuestionario (Brentari \& Golia, 2007). Existen muchos procedimientos para determinar la dimensionalidad de una prueba, algunos de los cuales se refieren a técnicas que se emplean luego de administrarla a un grupo de personas, tales como el análisis de componentes principales de residuos y el análisis del ajuste de los ítems. Ambos valores son computados por el software Winsteps ${ }^{\circledR}$.

\section{Resultados}

\section{Funcionamiento Diferencial de Ítems}

El funcionamiento diferencial de ítems indica que un ítem funciona de manera diferente en grupos de personas cuya habilidad es igual pero que difieren en alguna característica y que se debe a un error sistemático en el proceso de medición que distorsiona, en la misma forma, las mediciones obtenidas con una prueba (Osterlind, 


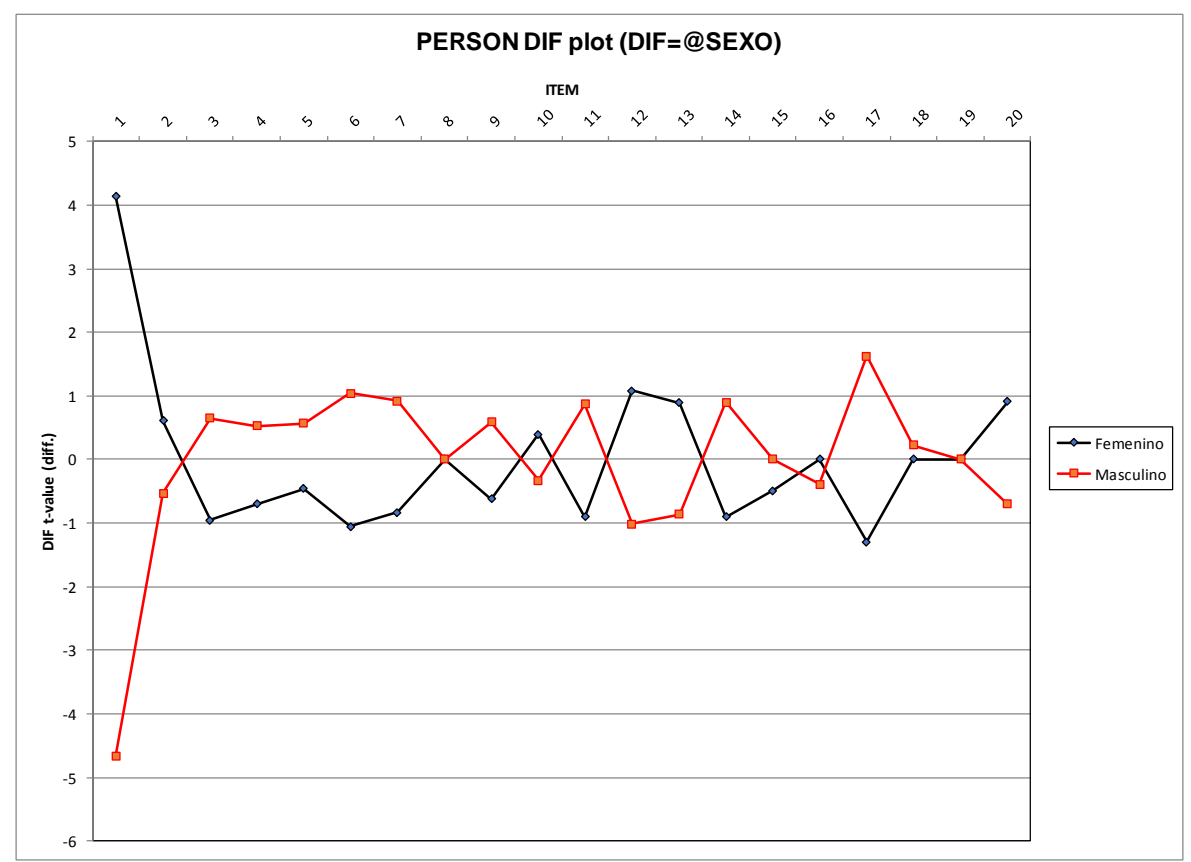

Figura 1. Valor t de la diferencia en el funcionamiento de los ítems. Valores superiores a $2.0 \mathrm{e}$ inferiores a -2.0 indican funcionamiento diferencial

1983). El software Winsteps ${ }^{\circledR}$ calcula el DIF con dos procedimientos diferentes: Mantel y regresión logística para preguntas politómicas, como las del cuestionario. El análisis se realizó tomando el sexo como variable para clasificar a los grupos.

En la Tabla 1, se presentan los estadísticos correspondientes a la detección de comportamiento diferencial. Se observa que el ítem 1 (ha ayudado a un desconocido a empujar su carro varado), cumple con las condiciones de un ítem con comportamiento diferencial, según el sexo. Linacre (2017), sugiere interpretar que un ítem presenta DIF, si los valores de diferencia son superiores a .5 y las probabilidades (p) de DIF son inferiores a .05. En la Tabla 1, el ítem 1 es el único que cumple con estas condiciones y por tanto funciona de manera diferente para hombres y mujeres.

Estos resultados se corroboran con los valores $\mathrm{t}$ del funcionamiento diferencial que aparecen en la figura 1. Los análisis posteriores no incluyen el ítem 1 que fue excluido por presentar funcionamiento diferente entre hombres y mujeres.

También se hizo un análisis de funcionamiento diferencial por edades, sin que se encontraran ítems que presentaran DIF.

\section{Confiabilidad}

La confiabilidad de Rasch fue de .84 con un índice de separación igual a 2.32. George y Mallery
(2003) establecen que los índices de confiabilidad superiores a .80 indican buena consistencia interna. Linacre (2017) plantea que un índice de separación como el obtenido (2.32) implica la conformación de por lo menos 3 grupos de personas, que para este estudio es aceptable.

\section{Dimensionalidad}

El análisis de componentes principales de residuos arrojó un valor propio de 2.20 para la varianza no explicada del primer contraste, que corresponde al $6.2 \%$. La varianza explicada por los ítems es de $33.6 \%$. Los ítems que tienen una mayor carga en el primer contraste son: 4, 6, 7, 12 y 15 (cargas superiores a .3 en valor propio). Los valores de ajuste de los ítems obtenidos y que corresponden a los valores de cuadrados mínimos (Linacre, 2017), son los presentados en la Tabla 2.

El valor esperado del ajuste es de 1.0 en cada ítem. Si es superior a 2.0 indica ruido en la medición o presencia de una dimensión diferente a la del constructo medido por la prueba. Los valores encontrados indican buen ajuste de los datos al modelo para los 19 ítems restantes. Adicionalmente, los coeficientes de correlación biserial puntual se ubican en valores aceptables, entre $.41 \mathrm{y} .56$, mientras los valores de discriminación están cerca de 1.0, siendo el más bajo .70 . 
Tabla 2. Indicadores de los ítems en el Modelo de Rasch

\begin{tabular}{lcccccc}
\hline Ítem & Dificultad & Error de medición & Ajuste próximo & Ajuste lejano & Discriminación & Biserial-puntual \\
\hline ALT02 & -.84 & .08 & .96 & .96 & 1.27 & .51 \\
ALT03 & .49 & .07 & .93 & .90 & 1.11 & .57 \\
ALT04 & .64 & .07 & .88 & .92 & 1.11 & .58 \\
ALT05 & -.29 & .08 & .97 & .98 & 1.20 & .52 \\
ALT06 & -.34 & .07 & 1.05 & 1.07 & .93 & .50 \\
ALT07 & .64 & .07 & .97 & 1.02 & .90 & .52 \\
ALT08 & .45 & .07 & 1.14 & 1.3 & .63 & .45 \\
ALT09 & -.25 & .07 & .94 & .93 & 1.22 & .56 \\
ALT10 & -.95 & .08 & .86 & .86 & 1.22 & .59 \\
ALT11 & -.24 & .08 & 1.01 & 1.00 & 1.10 & .50 \\
ALT12 & 1.53 & .09 & 1.07 & 1.22 & 1.02 & .41 \\
ALT13 & -.33 & .06 & 1.12 & 1.10 & .70 & .49 \\
ALT14 & .62 & .07 & 1.04 & .99 & .97 & .50 \\
ALT15 & .34 & .07 & 1.11 & 1.06 & .94 & .49 \\
ALT16 & -1.08 & .07 & 1.13 & 1.10 & .88 & .43 \\
ALT17 & .49 & .07 & .92 & .91 & .76 & .48 \\
ALT18 & -.5 & .07 & .98 & .97 & 1.11 & .58 \\
ALT19 & -.87 & .08 & .96 & .99 & 1.01 & .51 \\
ALT20 & .49 & .07 & & & \\
\hline
\end{tabular}

\section{Niveles de altruismo}

Se utilizó el procedimiento de anclaje de escalas (Bertrand \& Dupuis, 1989) para identificar ítems en distintos niveles de la escala. Se encontraron tres puntos de anclaje. En el punto uno, anclaron los ítems 2, 10, 16 y 19; en el punto dos los ítems $3,8,15,17$ y 20, y en el punto tres el ítem 12. Con estos tres puntos de anclaje se desarrolló una escala de niveles que clasificó a los participantes en cuatro niveles de desempeño, desde el que hace referencia al desempeño más bajo de altruismo (denominado A), hasta el nivel más alto de desempeño (denominado D). En la Tabla 3 se encuentra la distribución en estos niveles de los 244 participantes.

Los niveles de desempeño se relacionan con la escala de medición al establecer los límites inferior y superior de cada nivel de desempeño con la metodología planteada por Pardo (2010), en la que se utilizan los puntos de anclaje de preguntas con lo que se establece la descripción de los desempeños descritos en cada nivel.

Tabla 3. Distribución de los participantes en los niveles de altruismo

\begin{tabular}{lcccc}
\hline & \multirow{2}{*}{ Nivel } & $\begin{array}{c}\text { Cantidad de } \\
\text { participantes }\end{array}$ & Porcentaje & \multicolumn{2}{c}{ Puntos de corte } \\
\cline { 4 - 5 } & & $\begin{array}{c}\text { Límite } \\
\text { inferior }\end{array}$ & $\begin{array}{c}\text { Límite } \\
\text { superior }\end{array}$ \\
\hline $\mathrm{A}$ & 116 & 47.54 & & $<-0.43$ \\
$\mathrm{~B}$ & 121 & 49.59 & $>-0.43$ & $<=0.89$ \\
$\mathrm{C}$ & 6 & 2.46 & $>0.89$ & $<=2.01$ \\
$\mathrm{D}$ & 1 & 0.41 & $>2.01$ & \\
\hline
\end{tabular}

\section{Discusión}

En este estudio se analizaron las propiedades de la SRAS, utilizando el modelo de respuesta al ítem desarrollado por Rasch y se avanzó en la adaptación de la prueba, partiendo del trabajo previo de traducción y cálculo de fiabilidad hecho en Colombia (Aguilar-Pardo y Martínez-Cotrina, 2016).).

El análisis diferencial de ítems mostró que la prueba funciona mejor sin el ítem referido a haber ayudado a un desconocido a empujar un automóvil, por lo que en los análisis subsiguientes se prescindió de él. En Colombia, por razones preponderantemente culturales, no es común que las mujeres se involucren en comportamientos de ayuda de este tipo, que incluyen un alto grado de fuerza física. Debido a que las nevadas son una condición excepcional en Colombia, este ítem fue adaptado de la prueba original que planteaba la situación de ayudar a un desconocido a sacar el auto de la nieve. Esto subraya la necesidad de incluir, además de procesos de traducción y adaptación lingüística, la contextualización y adaptación cultural de la prueba en su conjunto.

La nueva evidencia sobre la validez de la prueba, proporciona información que permite entender que la SRAS es unidimensional. El valor propio del primer contraste es el esperado para el tamaño de la muestra y el número de ítems de la prueba (Linacre, 2009; Raîche, 2005). Adicionalmente, el conjunto de datos de los ítems, incluyendo los valores de correlación biserial 
puntual y de discriminación, indican un ajuste apropiado. Sin embargo, aunque se cumple con los criterios establecidos para establecer la existencia de una variable latente, nuevos estudios podrían analizar las posibles subdimensiones (o factores) que conforman la prueba.

En su estudio acerca de las características de la SRAS, Chaplain (2017) identificó tres factores que agrupaban los ítems de acuerdo con el riesgo que representaba la conducta altruista expresada en cada ítem. Si bien el análisis factorial exploratorio puede utilizarse para conocer la estructura de una variable latente, es discutible que los diferentes niveles de riesgo puedan considerarse verdaderos factores. La descripción de Chaplain se ajusta mejor a la mayor o menor probabilidad de ocurrencia de una conducta altruista, de acuerdo con el riesgo que representa para quien la produce.

En este estudio no se confirmó lo hallado por Chaplain (2017). En este caso la aplicación del modelo de Rasch dio lugar al hallazgo de tres anclajes, que podrían corresponder a cuatro niveles de altruismo, siendo el primero de ellos caracterizado por conductas que no alcanzan el grado de conformidad con la expectativa de comportamiento cívico básico, de acuerdo con el estándar nacional. No llegan a este nivel las personas poco proclives a dar indicaciones en la calle o a ceder el asiento en el autobús a un desconocido. En el segundo nivel de altruismo, estarían las personas que hacen lo que se describe en los ítems del primer nivel de anclaje. En el tercero, quienes además de lo anterior, contribuyen con entidades de caridad y dan otras muestras de prosocialidad, como cambiar billetes a desconocidos. El cuarto nivel de altruismo estaría configurado por las personas que además de hacer todo lo anterior, serían propensas a llevar a desconocidos en su automóvil. De acuerdo con los resultados obtenidos, este comportamiento sería el que marca el nivel más alto de altruismo en Colombia. Aunque es escasa la distribución de los casos de la muestra en los niveles más altos, es de esperarse que haya pocas personas en los escalones más altos de altruismo. Sin embargo, los niveles que se plantean, podrán ser explorados en nuevos estudios, para establecer si diferencian apropiadamente distintos grados de altruismo en la población.
Este estudio constituye un avance en la adaptación de la SRAS, no obstante, las propiedades de la escala podrían estar afectadas por cuestiones del entorno que ameritarían una adaptación más contextualizada. Por ejemplo, en Colombia la tenencia de automóviles depende del estrato socioeconómico de las personas. Tomando como base las estadísticas oficiales hay 0.14 autos por cada habitante entre 15 y 79 años (Mintransporte, 2017). Así, la probabilidad de llevar a un extraño en el automóvil podría depender más de la tenencia de vehículos, que de la propensión altruista. Asimismo, en el área rural del país son prácticamente inexistentes los ascensores, por lo que es baja la probabilidad de que alguien detenga la puerta del elevador a un desconocido.

Por lo anterior, se considera que hay evidencia para sustentar la existencia del altruismo como un rasgo estable, que puede ser medido a través de la SRAS. Sin embargo, nuevos estudios podrían ir más a fondo en el ajuste de los ítems que conforman la escala, logrando una mejor adaptación a las condiciones de vida en el contexto colombiano. Aun en el caso de instrumentos con alto grado de reconocimiento y utilización internacional, es importante y necesario realizar estudios acerca de las cualidades psicométricas en contextos específicos (López, González, \& Tejada, 2017).

Abordar el altruismo es importante por el constructo en sí, también por sus implicaciones en diferentes asuntos como la cohesión social en tiempos de inestabilidad social, política y económica, la violencia y la inseguridad (Igwe et al., 2020; Rious \& Cunningham, 2018; Taylor \& Hanna, 2018)

\section{Referencias}

Abello-Luque, D., Cortes-Peña, O., GarcíaMontaño, E., García-Roncallo, P., \& NietoBetancourt, L. (2017). Escala Multidimensional de Trastornos Afectivos, EMTA: Generación de indicadores psicométricos y escalas normativas de uso clínico. Revista Iberoamericana de Diagnóstico y Evaluación - e Avaliação 
Psicológica,

43(1),

187-198.

https://doi.org/10.21865/RIDEP43_187

Aguilar-Pardo, D., \& Martínez-Cotrina, J. (2016). Validation of the Self-report altruism scale test in Colombian University Students. Ánfora, 23(41), 17-35.

Arnocky, S., Piché, T., Albert, G., Ouellette, D., \& Barclay, P. (2017). Altruism predicts mating success in humans. British Journal of Psychology, 108(2), 416-435. https://doi.org/10.1111/bjop.12208

Ato, M., López-García, J. J., \& Benavente, A. (2013). Un sistema de clasificación de los diseños de investigación en psicología. Anales de Psicología, 29(3). https://doi.org/10.6018/analesps.29.3.178511

Auné, S. E., Blum, D., Abal, F., Lozzia, G., \& Horacio, F. (2014). La conducta prosocial: Estado actual de la investigación. Perspectivas en Psicología, 11(2), 21-33.

Bartholomeu, D., Montiel, J. M., \& Machado, A. A. (2016). Optimization rating scales for an athletes Socialization Scale by Rasch model. Universitas Psychologica, 15(4). https://doi.org/10.11144/Javeriana.upsy154.orsa

Berger, C., Cuadros, O., Rasse, C., \& Rojas, N. (2016). Diseño y validación de la Escala de Creencias Normativas Sobre la Prosocialidad en Adolescentes Chilenos. Psykhe (Santiago), 25(1), 1-17. https://doi.org/10.7764/psykhe.25.1.692

Brentari, E., \& Golia, S. (2007). Unidimensionality in the Rasch model: How to detect and interpret. Statistica, 67, 253-261. https://doi.org/10.6092/issn.1973-2201/3508

Chaplain, J. (2017). Altruism and volunteering among high school students: A mixed methods study [Colorado State University]. https://dspace.library.colostate.edu/bitstream/ handle/10217/181415/Chaplain_colostate_005 3A_14144.pdf?sequence=1

Chou, K.-L. (1996). The Rushton, Chrisjohn and Fekken Self-Report Altruism Scale: A Chinese translation. Personality and Individual Differences, 21(2), 297-298. https://doi.org/10.1016/0191-8869(96)000402

DeVellis, R. F. (2017). Scale Development: Theory and Applications (4th ed). Sage.
George, D., \& Mallery, P. (2003). SPSS for Windows Step by Step: A simple guide and reference. 11.0 Update (4th Edition). Allyn \& Bacon.

Gliem, J. A., \& Gliem, R. R. (2003, enero 1). Calculating, interpreting, and reporting cronbach's alpha reliability coefficient for likert-type scales. In Midwest Research to Practice Conference in Adult. Midwest Research-to-Practice Conference in Adult, Continuing, and Community Education, Columbus, Ohio.

Guarnaccia, C., Giannone, F., Falgares, G., Ozino Caligaris, A., \& Sales-Wuillemin, E. (2016). Differences in social representation of blood donation between donors and non-donors: An empirical study. Blood Transfusion. 14(6): 490-499 https://doi.org/10.2450/2015.0048-15

Igwe, P. A., Ochinanwata, C., Ochinanwata, N., Adeyeye, J. O., Ikpor, I. M., Nwakpu, S. E., Egbo, O. P., Onyishi, I. E., Vincent, O., Nwekpa, K. C., Nwakpu, K. O., Adeoye, A. A., Odika, P. O., Fakah, H., Ogunnaike, O. O., \& Umemezia, E. I. (2020). Solidarity and social behaviour: How did this help communities to manage COVID-19 pandemic? International Journal of Sociology and Social Policy, ahead-of-print(ahead-ofprint). https://doi.org/10.1108/IJSSP-07-20200276

Jerosch-Herold, C., Chester, R., \& Shepstone, L. (2017). Rasch model analysis gives new insights into the structural validity of the QuickDASH in patients with musculoskeletal shoulder pain. Journal of Orthopaedic \& Sports Physical Therapy, 47(9), 664-672. https://doi.org/10.2519/jospt.2017.7288

Kawamura, Y., \& Kusumi, T. (2018). The relationship between rejection avoidance and altruism is moderated by social norms. Personality and Individual Differences, 129, 2427. https://doi.org/10.1016/j.paid.2018.02.038

Khanna, R., Singh, P., \& Rushton, J. P. (1993). Development of the Hindi version of a SelfReport Altruism Scale. Personality and Individual Differences, 14(1), 267-270. https://doi.org/10.1016/0191-8869(93)90202E

Leimgruber, K. L. (2018). The developmental emergence of direct reciprocity and its 
influence on prosocial behavior. Current Opinion in Psychology, 20, 122-126. https://doi.org/10.1016/j.copsyc.2018.01.006

Linacre, J. M. (1997). KR-20 / Cronbach Alpha or Rasch Person Reliability: Which tells the "truth"? Rasch Measurement Transactions. Rasch.org.

https://www.rasch.org/rmt/rmt1131.htm\#: :tex $\mathrm{t}=\mathrm{KR} \% 2 \mathrm{D} 20 \% 20$ (Cronbach\%20Alpha), meas ures $\% 20$ underlying $\% 20$ these $\% 20$ simulated $\%$ 20data.\&text=For\%20inference $\% 20$ beyond $\%$ 20the\%20test,more\%20conservative $\% 20$ and $\% 201$ ess \%20misleading.

Linacre, J. M. (2009). WINSTEPS Rasch measurement computer program. Winsteps.com.

Linacre, J. M. (2017). WINSTEPS Rasch measurement computer program. Winsteps.com.

Liu, B., Huang, Z., Xu, G., Jin, Y., Chen, Y., Li, X., Wang, Q., Song, S., \& Jing, J. (2016). Altruistic sharing behavior in children: Role of theory of mind and inhibitory control. Journal of Experimental Child Psychology, 141, 222-228. https://doi.org/10.1016/j.jecp.2015.09.010

López, J. A., González, R. A., \& Tejada, J. M. (2017). Propiedades psicométricas de la versión en español de la escala de calidad de vida WHO Gol Bref en una muestra mexicana. Revista Iberoamericana de Diagnóstico y Evaluación - e Avaliação Psicológica, 44(2), 105-115. https://doi.org/10.21865/RIDEP44.2.09

Mislevy, R. J., Almond, R. G. and Lukas, J. F. (2003) A brief introduction to evidencecentered design. Research Report RR-03-16. Princeton, NJ: Educational Testing Service.

Molenaar, I. (1995). Some background for Item Response Theory and the Rasch Model. En G. Fischer \& I. Molenaar (Eds.), Rasch Models Foundations, Recent Developments, and Applications (pp. 3-14). Springer - Verlag.

Osterlind, S. J. (1983). Test item bias. Sage Publications.

Ovejero Bernal, A. (2007). Las relaciones humanas. Psicología social teórica y aplicada. Biblioteca Nueva.

Pardo, C. (2010). Manual para el procesamiento y análisis de datos aplicación piloto. En
UNESCO (Ed.), Compendio de los manuales del SERCE (pp. 225-253). UNESCO.

Raîche, G. (2005). Critical eigenvalue sizes (variances) in standardized residual principal components analysis. Rasch Measurement Transactions, 19(1), 1012.

Rious, J. B., \& Cunningham, M. (2018). Altruism as a buffer for antisocial behavior for African American adolescents exposed to community violence. Journal of Community Psychology, 46(2), 224-237. https://doi.org/10.1002/jcop.21936

Rushton, P., Chrisjohn, R. D., \& Fekken, C. (1981). The altruistic personality and the selfreport altruism scale. Personality and Individual Differences, 2(4), 293-302. https://doi.org/10.1016/0191-8869(81)90084-2

Santamaría-García, H., González-Gadea, M. L., Di Tella, R., Ibáñez, A., \& Sigman, M. (2018). The interplay between sharing behavior and beliefs about others in children during dictator games. Journal of Experimental Child Psychology, 166, 451-464. https://doi.org/10.1016/j.jecp.2017.08.016

Spector, P. (1992). Summated rating scale construction. SAGE Publications, Inc. https://doi.org/10.4135/9781412986038

Taylor, L. K., \& Hanna, J. R. (2018). Altruism born of suffering among emerging adults in Northern Ireland. Journal of Aggression, Conflict and Peace Research, 10(3), 157-169. https://doi.org/10.1108/JACPR-01-2017-0271

Velásquez, E., Martínez, M. L., \& Cumsille, P. (2004). Expectativas de autoeficacia y actitud prosocial asociadas a participación ciudadana en jóvenes. Psykhe, 13(2), 85-98.

Wittek, R., \& Bekkers, R. (2015). Altruism and prosocial behavior, sociology of. En International Encyclopedia of the Social \& Behavioral Sciences (pp. 579-583). Elsevier. https://doi.org/10.1016/B978-0-08-0970868.32158-4 